\title{
Machine Learning of Generalized Document Templates for Data Extraction
}

\author{
Janusz Wnek \\ Science Applications International Corporation \\ 1953 Gallows Road, Vienna, VA 22182, U.S.A. \\ Janusz.Wnek@saic.com
}

\begin{abstract}
The purpose of this research is to reverse engineer the process of encoding data in structured documents and subsequently automate the process of extracting it. We assume a broad category of structured documents for processing that goes beyond form processing. In fact, the documents may have flexible layouts and consist of multiple and varying numbers of pages. The data extraction method (DataX) employs general templates generated by the Inductive Template Generator (InTeGen). The InTeGen method utilizes inductive learning from examples of documents with identified data elements. Both methods achieve high automation with minimal user's input.
\end{abstract}

\section{Introduction}

Understanding documents is a relatively easy task for an intelligent human reader most of the time. This is due to the fact that the documents are prepared using some common assumptions about structuring them, and authors' intend to convey information in ways allowing readers accurate and efficient interpretation. The problem is, to what degree is it possible to reverse engineer the process of encoding data in documents and subsequently automate the process of extracting the data.

The main objective for creating most documents is to communicate visual information. Characters, combined in words, sentences, and paragraphs, have been intentionally placed on documents. We have found that a fundamental utility for reverse engineering of the process of encoding data in documents is provided by optical character recognition (OCR). Specifically, OCR-based features, i.e. characters, make ultimate sense in recognizing textual constructs, as opposed to other features, such as bounding boxes resulting from connected component analysis, frequency/distribution profiles, texture analysis, and other image processing artifacts.

Based on recognized character contents and its layout one can build even more meaningful constructs, such as words and phrases, and relate them with each other. We understand that this process is a subsequent, important element in reverse engineering the document encoding process. Words and phrases become elementary symbols in machine document processing. Understanding their relative usage enriches document representation. Now, given the set of symbolic elements related to each other via a set of defined relations, an intelligent system can learn patterns in their

D. Lopresti, J. Hu, and R. Kashi (Eds.): DAS 2002, LNCS 2423, pp. 457-468, 2002.

(c) Springer-Verlag Berlin Heidelberg 2002 
arrangement. Generalized pattern descriptions can be induced from exemplary definitions of data elements on some instance documents.

Generalization is a powerful knowledge transformation that allows learning of general descriptions that govern data element arrangement. The descriptions of symbol arrangements become the underlying representation for document structures. They disclose intentions of document authors with regard to data composition.

Fig. 1 illustrates the process of reverse engineering a document design as specified in the InTeGen method. It is assumed that each document file consists of ordered images of single pages, assembled in a prior process. One at a time, a template is "learned" for each document type. In the first two steps, the document image is extended with a symbolic description of its contents; i.e., with document text and relations between identified visual/textual constructs. Given the document image and OCR'd text, a user defines data elements on the image. This procedure involves drawing boundaries around the character strings constituting the data elements.

Data element (DE) definitions communicate user's goals for processing a particular document type at the moment. Refining goals can be realized by adding or removing definitions at a later time. Next, the symbolic representations of the document and data element definitions are input to a learning module. Given the first instance of a document, the symbolic information is simply stored in a document template. Processing the following instances involves assimilation into the template. More specifically, common textual patterns are found in both the new document and the current template, and the general patterns are stored as a new template description.

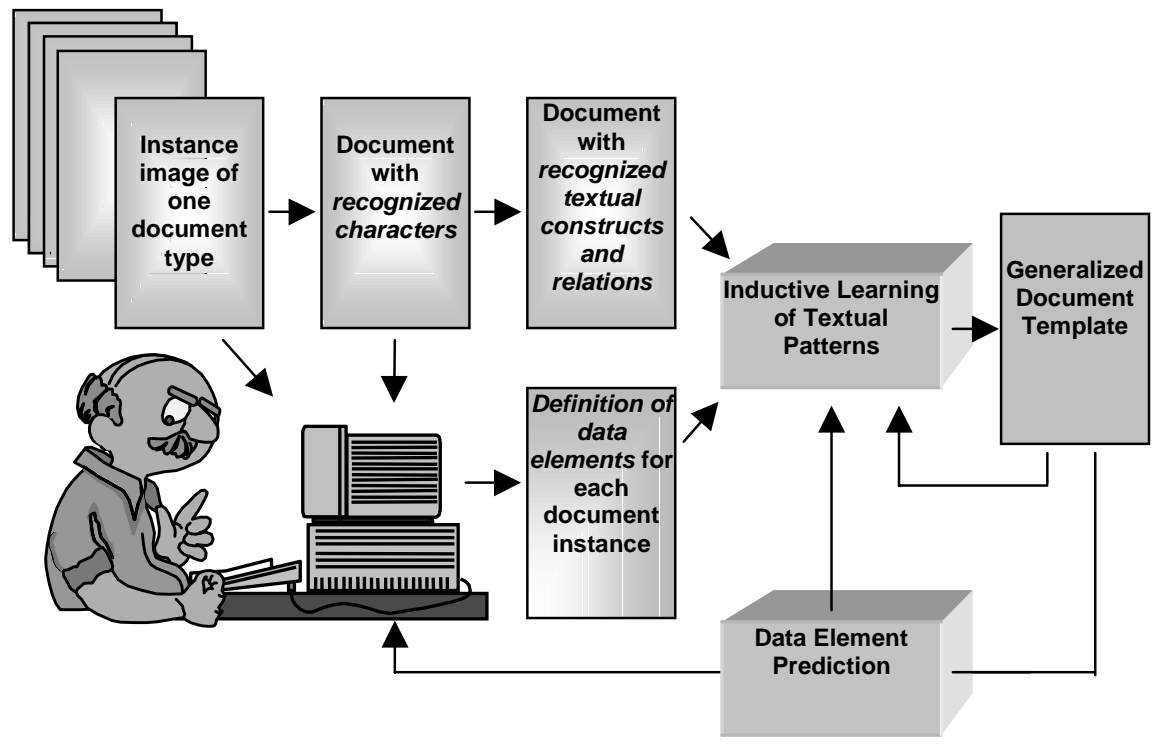

Fig. 1. Reverse engineering the document design process: Inductive Template Generator InTeGen

After each incremental template refinement, the InTeGen process is bootstrapped with the newly refined template. The template is utilized in predicting data elements of unprocessed training documents. First, the predicted data elements serve in 
assisting the user when defining data elements on new documents of the same type. Second, the predicted data elements are utilized in evaluating capabilities of the current template, before the decision about assimilating new documents into the template is made.

The Generalized Document Template represents the original design of one document type with regard to the guidelines provided by the user in the form of data element definitions. Accurate formulation of the hypothesis depends on consistency in specification of data elements across the document type and the user definitions of data element boundaries.

In summary, the goal of the combined InTeGen-DataX system is to automatically "learn" complex document structures, store them in general templates, and utilize them in a data extraction process. Reverse engineering of a document design is realized by a combination of optical character recognition, reconstruction and understanding of document components, and relating them to original intend by utilizing user's input.

\section{Inductive Template Generator: Document and Data Structures}

Historically, document conversion systems have focused on two major types of applications: full-text databases and forms processing. Examples of full-text applications include correspondence tracking and litigation support. Major forms processing applications (domains) include health care forms, tax forms, and the U.S. Census. There is a large class of applications that lies between these two major types. These applications involve processing flexible forms. Examples include bills of lading, invoices, insurance notifications and mortgage documentation. Such applications typically are characterized by a large number of document variants. Today, most forms processing applications are designed to recognize and capture data from a small number of form types. Even the most versatile operations typically process at most a few dozen forms. The flexible form applications, in contrast, typically involve hundreds to thousands of document variants.

In order to facilitate processing of flexible form documents, a generalized document template is introduced to represent a generalized document model. The generalized document template captures characteristics of the complete document as opposed to page (or form) templates that generalize single pages only. A generalized document template contains generalized descriptions of data elements. A data element is a logical unit that defines an object for extraction. We assume that data elements contain one or more lines of textual information and can be enclosed in a rectangular bounding box. The bounding box provides necessary grouping of all words relevant to the data element. In the remainder of the paper we refer to the generalized document template simply as the template.

Defining a data element is a very simple operation which involves drawing a bounding box around the data element and selecting a predefined name for that data element (Fig. 2). The data element description and a necessary context are both acquired from the document and maintained automatically. Keywords, key phrases, or the layout of a data element on a page can provide the context. 
Data extraction from new documents, i.e. documents not used for training is based on the application of the template. The context enables finding the data element on the document and helps in determining the scope of the data element.

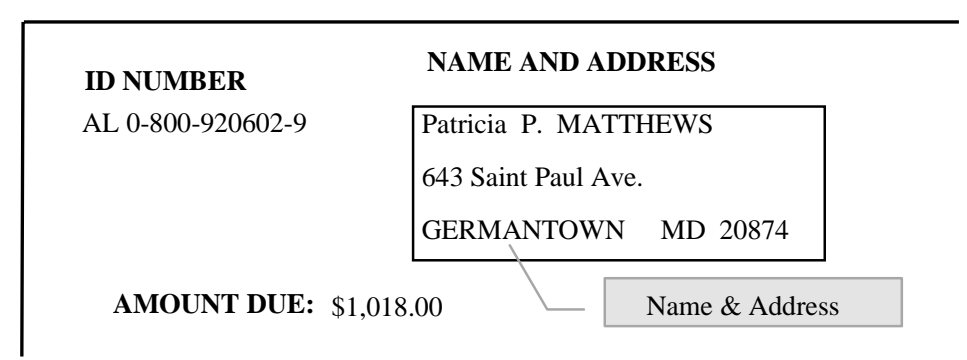

Fig. 2. A document fragment with a user-defined data element.

In the template generation process, a document is described at several levels of abstraction: page, line, word, and character levels. Page level consists of descriptions of all pages included in the document. These are simply page dimensions and margin offsets. Line level consists of the total number of document lines; vertical offsets to line bases from the beginning of the document, and numbers of words in each line. An OCR engine predetermines division of a document into lines. Word level consists of the total number of document words, horizontal offsets to words' start and end measuring from the line beginning, and number of characters in each word. Character level consists of the total number of document characters, horizontal offsets to characters, their widths and heights, as well as the complete document text.

A template represents a generalized document description. In the template, each data element is represented by a list of alternative descriptions. Alternative descriptions represent various patterns in DE contexts that are significantly different. For example, if documents from the same class use different captions to tag the same data element then the system may use two or more alternative descriptions to represent the changing captions.

A single description contains four attribute types: physical, lexical, contextual, and control type.

Physical attributes include ranges of vertical and horizontal positions in the document, width range, height range, maximal width and height including white space available. Positions, widths, and heights are measured in pixels. Other physical attributes include number of lines, word position in the line from both left and right, number of words in the line, and number of characters in the first word.

Lexical attributes correspond to document description at the three levels: line level, word level and character level. Line level consists of the total number of DE lines; vertical offsets to line bases from the beginning of each DE, and numbers of words in each DE line. Word level consists of the total number of DE words, horizontal offsets to words' start and end from DE line beginning, and number of characters in each word. Character level consists of the total number of DE characters, horizontal offsets to characters, their widths and heights, as well as the complete DE text. The text consists of words separated by single spaces. Lexical description provides an example of data element contents. It does not contain any generalized information. 
Contextual attributes include number of words in the context, and actual words with distance and utility measurements. The words are naturally ordered in a sequence according to the line order produced by an OCR and read from left to right. The distances are grouped into two categories: pixel and word distances. Pixel distance describes span (in pixels) between the context word and the DE origin, i.e. the beginning of the first DE word. Two ranges in vertical (x) and horizontal (y) dimensions represent the pixel distance. Word distances are stated using the number of words between the context word and the first DE word. Two word distances are used: sequential and vertical. Sequential word distance counts words as they were read line by line, from left to right. Vertical word distance counts the number of lines containing words intersecting the column delineated by the width of the context word. For each vertical distance, the number of occurrences (frequency) in various documents is collected.

The distances are whole numbers. Positive numbers indicate that the context word is after the first DE word. Negative number indicates that the context word is before the first DE word. The order depends on the type of distance. For pixel distances, "before" means that the context word is above in vertical dimension and to the left in horizontal dimension. For sequential word distance, "before" means that the context word is closer to the beginning of the document than the DE word. For vertical word distance, "before" means that the context word is above DE.

The utility of a context word indicates the usefulness of the word for searching purposes. Four values are being assigned: best, good, fair, and poor. Best utility is assigned to words that precisely identify a DE by themselves and are unique. Good utility is assigned to words that precisely identify a DE (in terms of the search method, e.g. starting from the top of the document) but are not unique. Fair utility is assigned to unique words that occasionally may fail the search. Poor utility is assigned to words that should not be used in searches.

Table 1. Context word utility with regard to potential search precision and uniqueness within the document.

\begin{tabular}{|c|c|c|}
\hline Utility & Search Precision & Uniqueness \\
\hline Best & + & + \\
\hline Good & + & - \\
\hline Fair & - & + \\
\hline Poor & - & - \\
\hline
\end{tabular}

Control attributes include word types for selected DE words (e.g. first words in each DE line), indices of the first DE line, word, and character; generalization counter, and DE identifier in the domain. Word types categorize words into several classes, such as: numeric with various combinations of related characters (e.g., +, -, \#, \%, etc.), alphabetic (ALL CAPS, all lower case, mixed, etc.), alphanumeric, dates, etc. Generalized word types may have one or more values. 


\section{Inductive Template Generator: The Method}

A template for a given document class is built in an incremental way from examples of documents (training documents) and data element definitions. The documents are first assembled from scanned and OCR'd pages, and stored in the document description data structure. For each document, a user defines a set of data elements by drawing bounding boxes on the document image. At the minimum, DE definitions consist of five numbers only: vertical and horizontal position of the bounding box origin, width, height, and data element identifier.

\subsection{Induction}

The procedure for generalizing templates iterates through the list of data element definitions and generalizes each data element description. Generalization of DE descriptions is performed independently of each other.

Given the first training document and a set of data element definitions, InTeGen initializes the template by copying the document description to the template description, and by constructing and attaching initial data element descriptors to the template for each data element. Each DE descriptor is constructed based on its definition and document contents: filling out the descriptor involves extraction of relevant lexical descriptors from the document description, determining values of physical and control attributes, and constructing contextual attributes. Initially, contextual attributes comprise all document words. For each attribute, all types of distance measurements are taken, i.e. pixel distance, sequential and vertical word distances. Copying the document description to the template is simple because both document and template descriptions share the same data structures.

Given the next training document and the set of DE definitions, generalization with the template occurs unconditionally. The following training documents may or may not be generalized with the template depending on template generality, or in other words, the template's capability of correctly predicting the place and scope of its data elements. Template generalization equates to generalization of any of the DE descriptions. In order to determine if a template covers DE descriptions from the training document, a prediction test is conducted for each DE. The template's DE description is generalized only if it is not capable of correctly predicting the given DE on the training document.

DE description generalization involves generalization of particular attributes. For physical and control attributes expanding the range of measured values is sufficient. The selection of this type of generalization was dictated by the nature of document attributes, values of which are continuous rather than discrete. For example, assuming that DE sequential word distances for the first two documents were 5 and 8 , the generalized distance results in a range between 5 and 8 . This means that if the third document distance value is 7 then the third document is covered by that condition.

Generalization of context attributes involves intersecting two sequences of words and then, for the common words, generalizing their values. For example, given the two sequences of words, T_CAi, D_CAi, below, describing contextual attribute (CA) of data element (i) on template (T) and document (D), their generalization results in T_CAi. Spacing between words was added to highlight the method of aligning words. 
Table 2. Generalization of two sequences.

\begin{tabular}{|l|l|l|l|l|l|l|l|l|l|l|}
\hline T_CAi: & w1 & & w2 & w3 & w4 & w5 & w6 & & w7 & w8 \\
\hline D_CAi: & w1 & w8 & w2 & & w4 & & w6 & w3 & w7 & w8 \\
\hline \hline T_CAi: & w1 & & w2 & & w4 & & w6 & & w7 & w8 \\
\hline
\end{tabular}

The method for aligning words incorporates three general preference criteria for selecting words for alignment. Additional criteria may be added based on document characteristics in a particular document domain. The general preference criteria include:

1. Words in the template are more significant then those in the document (unless the template was not generalized yet).

2. Alignment should maximize the number of common words.

3. Alignment should take into consideration keywords or key phrases delimiting identifiable sections of a document.

After DE generalization, the DE description is evaluated with regard to its utility. There are two criteria for testing DE description utility. The first is based on description evaluation, and the second is based on performance evaluation. If both tests are passed then the DE description replaces the previous one in the generalized template. If any of the tests fails, then a new alternative description is created.

Description evaluation involves determining the amount of change in context close to DE. The change is determined by comparing sequence word distances in the last two generalizations. If the distance to the word immediately proceeding DE or the distance to the word immediately following DE increased by more than predefined thresholds, then a new alternative rule is added. The threshold values are determined empirically for the given document-processing domain.

Performance evaluation involves testing prediction capability using the recently generalized DE description. The document that was recently used for generalization serves as a testing material. This procedure allows for producing consistent and complete descriptions with regard to the training set of documents.

In some cases a data element may show a high degree of variability in addition to not having precisely defined boundaries. For example, a data element may consist of a varied number of lines with some other data immediately following it. The attached data may easily be construed as an integral part of the DE especially when it takes place optionally. In order to detect ambiguous boundaries, InTeGen learns the structure of the edit phrases that delimit a data element.

The procedure for generalizing templates takes advantage of failing some prediction tests to learn the edit phrases. Edit phrases consist of the difference between the predicted text and the defined text. The phrases are generalized, stored and utilized when predicting data elements. The edit phrase data structure consists of a location attribute, a generalized text of the phrase, and a generalization counter. The generalized text of the edit phrase stores actual words if they reappear, or word types for varied phrase words. 


\subsection{Prediction}

Prediction plays an important role in building a generalized template (InTeGen) and actual data extraction from new documents (DataX). Given the fact that during template generation data element definitions are available from a user as an integral part of the training set, DE definitions may also serve as a basis for comparison with predicted values. Feedback produced that way is invaluable for an automated system. It provides a means for detecting the need for performing generalization, and once generalization is done, the verification of a template's utility. As a result of such verification, it may lead to follow-up actions such as building alternative descriptions.

Given data element id, the data element prediction procedure selects the DE description from the template. DE description is by itself disjunctive. It consists of alternative descriptions covering variations in the DE context observed on different training documents. The procedure has to decide which alternative description is best for making prediction on the given document. In order to do this, it performs partial prediction based on all of the alternative descriptions and then selects one to complete the prediction process. In the case where no alternative description can be successfully applied, the procedure reports failure.

The prediction process breaks down into two stages: finding the origin of the data element on the current document and determining its size. Finding the DE origin is simplified to finding the first word of the data element, which is facilitated by the DE description constructed in the learning phase. This task involves selecting the proper search phrase to address the first word, applying one of the distances to find candidate words, and determining the best candidate based on testing the first word properties. Once the first word is known, the origin of the DE bounding box is also known. Determining DE size is based again on measurements and characteristics gathered during the learning phase and stored in DE description.

Finding the DE origin consists of searches for the DE using context phrases located before or after the data element. Regularly, one of the searches succeeds in finding a reliable phrase that is located in consistent proximity from the data element that yields anticipated DE origin. If the two searches do not yield an anticipated DE origin, a range of words for examination is constructed, and the DE origin is decided based on sequential location in the target document and data type of the DE first word. If there is no valid context phrase before or after the DE, or it is not possible to predict using the sequence distance, context phrases above and under are examined, and the vertical distance may be applied to predict the DE origin.

In the process of finding DE origin, a procedure is employed to search for context phrases. Based on the template description, it constructs a multi-word context phrase that precisely addresses DE. The phrase has to be close to the DE to assure the most precise reconstruction of DE location. In order to assure that the context phrase is not mismatched within the currently processed document, a unique landmark in close proximity to the phrase is being used. The unique landmark is a word that was assigned the best search utility during template learning. The procedure returns the address of the word nearest to the DE origin. 


\section{Evaluation}

The InTeGen-DataX method has been implemented in the ML-FromForms system. The system is capable of processing variety of flexible form documents. The system integrates various image processing and document understanding components including, inductive document classifier for recognizing document types (Wnek, 1999). Fig. 3 presents a sample of documents that can be processed by MLFromForms (only first pages of multiple-page documents are shown). For some applications, templates for several hundred of document types have been created and data successfully extracted.
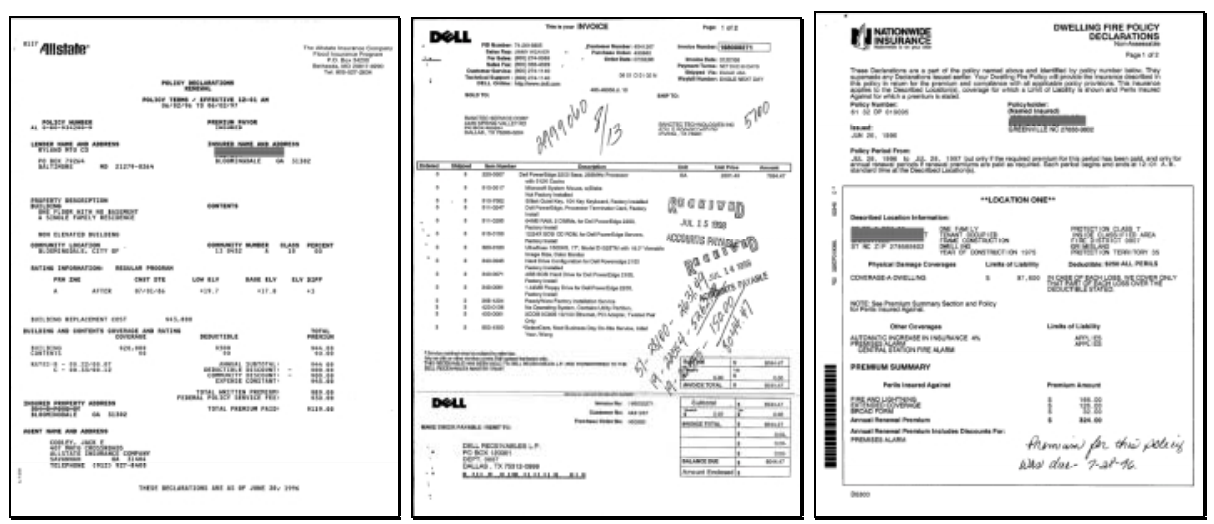

Fig. 3. Examples of insurance and invoice documents used for template training and data extraction. ML-FromForms is capable of extracting any machine-printed, data element identifiable on such pages.

The main objective for the testing was to establish prediction accuracy of the DataX method, i.e. accuracy of finding and extracting data elements from documents similar to those presented in Fig. 3. OCR accuracy was not of our concern, hence ground truth data was easy to create and consisted only of bounding boxes and identifiers of defined data elements. Another two objectives for testing was to establish approximate number of training examples required to achieve a desired prediction accuracy, and to confirm system's stability on known (training) data.

There may be many other factors that influence prediction accuracy in general. First of all, not all documents or their parts may qualify as having flexible layouts. Therefore, not all data elements may be extracted from them. Second, document image quality and OCR accuracy may influence both quality of data element prediction accuracy, as well as data element contents.

Our testing involved 10 selected document types for which ground truth data was created. For each document type up to 11 data elements were defined, totaling 103 data elements in all document types. Ground truth data was created for 20 documents in each type. In all, 2,060 data elements were defined. In each train-test session, the system was trained using from 1 to 20 training examples and evaluated using all 20 documents. 
Three error types were measured: omission error, commission error, and precision error. Table 3 defines the error types for various occurrences of defined and predicted pairs of data elements (DE). Precision errors capture imprecision in extracting data element, i.e. too small or too large bounding box. This error type is usually of lesser concern to users than the errors of commission or omission because it may be corrected by post processing.

Table 3. Error types used in determining data element prediction accuracy.

\begin{tabular}{|c|c||c|c|c|}
\hline DE defined & $\begin{array}{c}\text { DE } \\
\text { predicted }\end{array}$ & $\begin{array}{c}\text { Omission } \\
\text { Error }\end{array}$ & $\begin{array}{c}\text { Commission } \\
\text { Error }\end{array}$ & $\begin{array}{c}\text { Precision } \\
\text { Error }\end{array}$ \\
\hline Yes & Yes, exact match & 0 & 0 & 0 \\
\hline Yes & Yes, partial match & 0 & 0 & 1 \\
\hline Yes & Yes, no match & 1 & 1 & 0 \\
\hline Yes & No & 1 & 0 & 0 \\
\hline No & No & 0 & 0 & 0 \\
\hline No & Yes & 0 & 1 & 0 \\
\hline
\end{tabular}

Charts in Fig. 4 summarize DataX testing for 10 document types. The overall error rate includes omission and precision error rates only, because the system did not make any errors of commission. Majority of the errors are due to imprecise scope of bounding boxes. The system makes less than $5 \%$ errors (2\% omission errors) after learning from about 5 training examples. (Note that at 5 training examples, the system is still evaluated on 15 unknown examples, and maintains $5 \%$ error rate). In addition, system achieves $0 \%$ error rate when presented with all known documents, which suggests that the template descriptions are consistent and complete with the data.
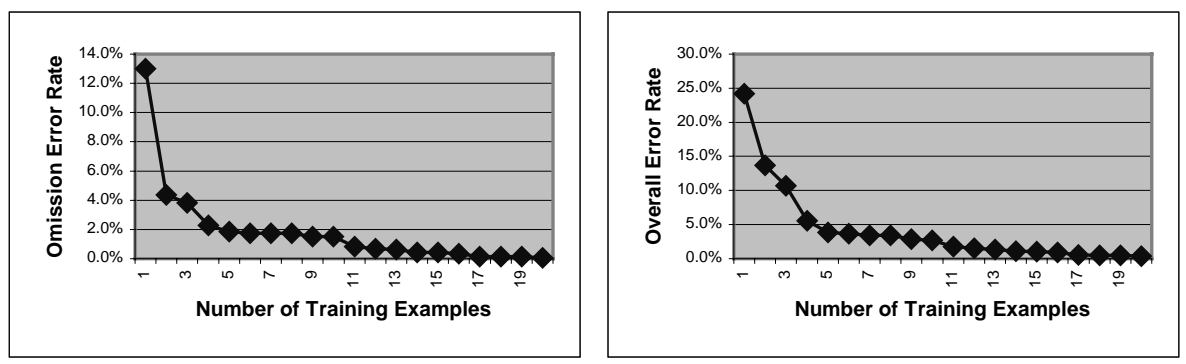

Fig. 4. Data element prediction accuracy.

\section{Related Research}

The document reverse engineering process may take many different forms and extent of automation. Some systems may have such knowledge preprogrammed, others, more flexible, may use some declarative representation for modeling document domains. Document models may be acquired in a variety of ways too. Some systems may require explicit rule coding for finding data elements. Others, through a graphical user interface, may support template design. Still, all data descriptions have to be detected and input by a user. Overall, application of form processing methods to 
complex documents may require manual or semi-manual preparation of complicated models, which makes them prohibitively expensive due to labor costs.

For example, Koppen et al. (1998) describe a system for an automated evaluation of invoices. The purpose of the system is to detect and recognize price entries of item tables in invoices. The system is not model-driven but it makes many assumptions about the structure of the invoices and data elements constituting a table. It does not attempt to extract other, non-table like date elements. Consequently, the system seems to be restricted to a very specific category of documents.

Bayer and Mogg-Schneider (1997) describe a more flexible system for processing invoices. The system consists of two components, OCR tool and data extraction component that contains declarative knowledge about the domain. The system uses special language for describing objects in structured documents. The drawback of the method is that document models have to be carefully crafted and described by a document engineer. In a selected domain of insurance invoices, the system achieved $50 \%$ automation rate with an error rate below $1 \%$ on selected data elements. Even though the system has a potential for processing diverse documents, the document definition process seems to be complex.

In contrast to those systems, the InTeGen method completely automates the process of document definition through machine learning. The method is applicable to a variety of flexible form documents, and provides easy and fast document definition. The only drawback of the method, the necessity of defining models for each document type, is offset by the simple and expeditious document definition process.

In a broader sense, the InTeGen method is related to the automated discovery of logical structure in text documents (Esposito et al., 1994; Cesarini et al, 1999). The aim of automated discovery of logical structure is to create a hierarchy of logical components of a document from given physical instances of the document. Most of the methods target specific types of documents, such as technical papers or office documents. Each approach assumes some degree of prior knowledge of the style of a document.

Another fundamental distinction in various approaches stems from the relative roles of content and layout in the definition of a logical structure. A logical structure is more content-oriented if its definition relies more heavily on internal meaning. A layout-oriented structure relies more heavily on visual presentation. Therefore, in content-oriented methods, OCR data is analyzed and utilized; whereas, in layoutoriented methods, image-processing methods, such as segmentation, projection profiles, texture analysis, play the major role (Dengel, 1992, Summers, 1995).

\section{Conclusions}

Data extraction from context-form type documents is a difficult problem because of the indefinite structure of such documents. Data fields do not have fixed locations and characteristics on a page, and may flow between pages of documents. These documents are frequently beyond the capability of the currently commercially available forms processing systems, which require significant user involvement in the process of defining fields for extraction. The process requires competence and is time consuming. The user has to: (1) learn how to operate a new system, (2) discover patterns in data format, and (3) learn ways of transferring this knowledge to the 
document processing system. The third requirement may be extremely time consuming and often frustrating given the system constraints. Moreover, the user's input, calculations and estimations may often be imprecise and lead to system errors.

The InTeGen-DataX method offers several advantages over the earlier methods. The most important feature manifests itself in the minimal user's involvement in the process of defining fields for extraction. The user is only required to identify what the location of the object of extraction is. The user is not required to provide any additional characterization of the object. All necessary information needed to characterize the object and its context is acquired automatically. It minimizes human effort and chances for introducing errors. Additionally, the system supports data element definition process by predicting DEs based on currently defined templates. This feature greatly speeds up template development, and assures template quality.

The next advantage results from robust generalization of training examples. The example documents are real documents as opposed to synthetic document models. This way the system encounters not only variability in data arrangement but also possible noise. By processing and filtering out noisy descriptions, the system becomes immune to noise when processing subsequent documents. In addition, machine learning makes it feasible to include the template generation module in a real-time system, and acquire knowledge about new document cases during production. The input can be directed from key-from-image processing of failed documents. This creates opportunity for the document processing system to become instantly responsive to incoming variations in document designs.

Finally, the symbolic descriptive patterns are language independent. The method could be integrated with versatile OCR devices that process documents in different languages, e.g. Latin, Cyrillic, Greek, Arabic, Japanese, or Chinese.

\section{References}

Bayer, T., Mogg-Schneider, H., "A Generic System for Processing Invoices," Proc. Int. Conf. on Doc. Analysis and Recognition, pp.740-744, IEEE Computer Society Press, 1997.

Cesarini, F., Francesconi, E., Gori, M., and Soda, G., "A Two Level Knowledge Approach for Understanding Documents of a Multi-Class Domain," Proc. Int. Conf. on Doc. Analysis and Recognition, pp.135-138, IEEE Computer Society Press, 1999.

Dengel, A., "ANASTASIL: A System for Low-Level and High-Level Geometric Analysis of Printed Documents" in Structured Document Image Analysis, Springer-Verlag, Berlin, 1992.

Esposito, F., Malerba, D., and Semeraro, G., "Multistrategy Learning for Document Recognition," Applied Artificial Intelligence, Vol. 8, pp.33-94, 1994.

Koppen, M., Waldostl, D., and Nickolay, B., "A System for the Evaluation of Invoices," in Document Analysis Systems II, pp. 223-241, World Scientific, 1998.

Summers, K., "Near-Wordless Document Structure Classification," Proc. Int. Conf. On Document Analysis and Recognition, IEEE Computer Society Press, 1995.

Wnek, J., "Learning to Identify Hundreds of Flex-form Documents," Proc. of SPIE, Document Recognition and Retrieval VI, D. Lopresti and J. Zhou (Eds.), Vol. 3651, pp. 173-182, 1999. 